\title{
Research on Cooperative Transmission Based on Cross-layer Routing Design in Vehicular Ad-hoc Networks
}

\author{
Xuefei Zhang \\ School of Electronics and Information \\ Nantong University \\ Nantong, China \\ 916244965@qq.com
}

\author{
Guoan Zhang* \\ School of Electronics and Information \\ Nantong University \\ Nantong, China \\ *Corresponding author \\ gzhang@ntu.edu.cn
}

\begin{abstract}
The influence of cooperative transmission based on cross-layer routing design to the wireless vehicular Adhoc networks is studied. Firstly, the cross-layer cooperative transmission model is built, and then according to different quality of service requirements, The objective functions expressions of the optimal solutions for two addressed optimization problems are developed and later used as quantitative criteria of the routing decision. Simulation results show that the criteria using cooperative transmission typically yield more efficient routes than the comparable schemes in terms of end-to-end reliability and total transmission power.
\end{abstract}

Keywords-vehicular network;Ad-hoc network;cooperative transmission; cross-layer routing

\section{INTRODUCTION}

Vehicular Ad-hoc network is a special kind of mobile wireless ad-hoc network, which is based on a vehicle with a communication module for the nodes. Through wireless communication between the vehicle nodes to achieve the vehicle traffic information, traffic information spread quickly, you can discover and alleviate traffic congestion, effectively avoiding traffic accidents, and therefore has a very high prospect and research value[1]. In addition, the vehicle and the internet connection can also solve people's vehicle during the mobile office,multimedia entertainment needs.

Wireless vehicular Ad-hoc networks is highly dynamic, the network connection is interrupted frequently with multi-hop transmission[2], due to the above features, the traditional vehicle routing protocols are difficult to meet the requirements of information transmission in all aspects. The mobility of vehicles caused unpredictable communication topology and unstable communication channel condition can not be completely solved through traditional hierarchical network protocol systems[3]. Therefore, in order to meet QoS requirements, cross-layer routing design is needed to improve vehicle performance network communication by the interaction between the layers[4].

In this paper, we studied cooperative communication based on cross-layer routing design in wireless vehicular Ad-hoc networks, which can affect the performance of transmission. We constructed a cross-layer model of cooperative communication systems, and the study drew that collaborative communication mode can significantly reduce the cost of wireless links and the communication outage probability, whic improved the performance of vehicular networks.

\section{SYSTEM ARCHITECTURE}

\section{A. Physical Layer Model}

Using TDMA mechanism to build the physical layer model, TDMA mechanism has not competitive mechanism collision retransmission issues, data transfer is not equired too much control information, the node idle slots timely manner to sleep. TDMA mechanism requires strict time synchronization between nodes, so the message is transmitted simultaneously to the sink in the multiple sets of information source [5]. Assuming all nodes of wireless vehicle Ad-hoc network all nodes uniformly distributed within the designated area, and the model takes Rayleigh fading and shadowing fading and path loss and other factors into account, therefore, The wireless link between the source vehicle node $\mathrm{s}$ and the destination vehicle node $\mathrm{d}$ is modelled as:

$$
\partial_{s d}=\frac{c_{s d}}{d_{s d}^{\alpha / 2}}
$$

Require the operating modes of all vehicle nodes are full duplex mode, where $d_{s d}$ means the distance between vehicle node $\mathrm{s}$ to vehicle node $\mathrm{d}, \quad \alpha$ is the path loss exponent, generally ranging from 4 to $10, c_{s d}$ expressed the channel fading characteristics, required a obey zero mean and unit variance Gaussian variable, and independent and identically distributed.

\section{B. MAC Layer Communication Pattern}

Define a relay vehicle node $r$ to achieve collaborative communication of information, cooperative communication of MAC layer model divided into two stages in the time. In the first stage, the source node $\mathrm{s}$ transmit information to the destination vehicle node $d$ and the relay vehicle node $r$ in the form of broadcast message. In the second stage, the relay vehicle node $r$ will receive the forwarded message successfully decoded and transmit it to the destination vehicle node $\mathrm{d}$, if the information received is not successfully decoded, the relay vehicle node $r$ will not forward message, the source node s vehicle will resend the message. Figure 1 is Cooperative transmission system model in vehicular network. 


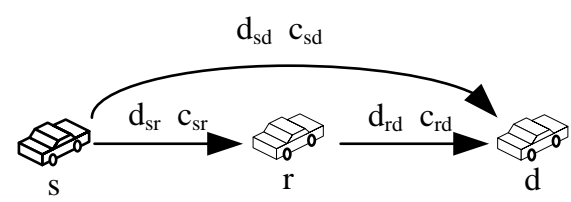

Figure 1. Cooperative transmission system model in vehicular network

\section{RESEARCH ON PERFORMANCE OF COOPERATIV TRANSMISSON AND SIMULATION}

Outage probability of point-to-point communication is used to reflect the reliability of inter-vehicle communication. Global transmission power is used to reflect the effectiveness of inter-vehicle communication. The advantages of collaborative communication is reflected by comparing with direct communication.

\section{A. Outage Probability of Cooperative Transmission}

Firstly we need derive the outage probability expressions of direct communication between the vehicles, define the maximum average mutual information of input and output between the source node and the destination node as[6]:

$$
I_{s d}^{D}=\log _{2}\left(1+S N R\left|\partial_{s d}\right|^{2}\right)
$$

Where $\partial_{s d}=\frac{c_{s d}}{d_{s d}^{\alpha / 2}}, S N R$ represents signal to noise ratio of communication.

Data rate is defined as $R$, unit is bits $/ s / H z$, use $I_{s d}^{D}<R$ to indicate that the communication interruption occurs in traffic, which is equivalent to:

$$
\left|\partial_{s d}\right|^{2}<\frac{2^{R}-1}{S N R}
$$

Due to follow Rayleigh fading, and follow the exponential distribution, so the inter-vehicle communication outage probability is:

$$
\begin{aligned}
P_{s d}^{D}=\operatorname{Pr}\left[I_{s d}^{D}\right. & <R]=\operatorname{Pr}\left[\left|\partial_{s d}\right|^{2}<\frac{2^{R}-1}{S N R}\right] \\
& =1-\exp \left(-\frac{2^{R}-1}{d_{s d}^{-\alpha} S N R}\right) \\
& \approx d_{s d}^{\alpha} \cdot \frac{2^{R}-1}{S N R}
\end{aligned}
$$

So that the outage probability expression of intervehicle direct communication is:

$$
P_{s d}^{D} \approx d_{s d}^{\alpha} \cdot \frac{2^{R}-1}{S N R}
$$

Based on the MAC layer communication mode, through the cooperative communication between vehicles, if the relay vehicle node can realize completely decoding information[7], it will forward the information to the destination vehicle node, if the relay vehicle node can't realize, the source vehicle node needs to resend this information, we define that information can eventually be decoded completely and the destination vehicle node can receive this information in this case. So considering these two cases we get the maximum average mutual information expression:

$$
\begin{aligned}
& I_{s d}^{F}=\frac{1}{2} \min \left\{\log _{2}\left(1+S N R\left|\partial_{s r}\right|^{2}\right),\right. \\
& \left.\log _{2}\left(1+S N R\left|\partial_{s d}\right|^{2}+S N R\left|\partial_{r d}\right|^{2}\right)\right\}
\end{aligned}
$$

Due to (2) and (6) we can obtain the maximum average mutual information of input and output between the source node and the destination node in the case of cooperative communication:

$$
I_{s d}^{C}= \begin{cases}\frac{1}{2} \log _{2}\left(1+2 S N R\left|\partial_{s d}\right|^{2}\right), & \left|\partial_{s r}\right|^{2}<g(S N R) \\ \frac{1}{2} \log _{2}\left(1+S N R\left|\partial_{s d}\right|^{2}+S N R\left|\partial_{r d}\right|^{2}\right),\left|\partial_{s r}\right|^{2} \geq g(S N R)\end{cases}
$$

Where $g(S N R)=\frac{2^{2 R}-1}{S N R}$, which is similar to the definition of the (3). Also use $I_{s d}^{C}<R$ to indicate the condition that interruption of cooperative communication occurs between vehicles, is equivalent to :

$$
\begin{aligned}
& \left(\left\{\left|\partial_{s r}\right|^{2}<g(S N R)\right\} \bigcap\left\{2\left|\partial_{s d}\right|^{2}<g(S N R)\right\}\right) \bigcup \\
& \left(\left\{\left|\partial_{s r}\right|^{2} \geq g(S N R)\right\} \bigcap\left\{\left|\partial_{s d}\right|^{2}+\left|\partial_{r d}\right|^{2}<g(S N R)\right\}\right)
\end{aligned}
$$

So thtat the outage probability of inter-vehicle cooperative communication can be expressed as follows:

$$
\begin{aligned}
P_{s d}^{C} & =\operatorname{Pr}\left[I_{s d}^{C}<R\right] \\
& =\operatorname{Pr}\left[\left|\partial_{s r}\right|^{2}<g(S N R)\right] \operatorname{Pr}\left[2\left|\partial_{s d}\right|^{2}<g(S N R)\right] \\
& +\operatorname{Pr}\left[\left|\partial_{s r}\right|^{2} \geq g(S N R)\right] \operatorname{Pr}\left[\left|\partial_{s d}\right|^{2}+\left|\partial_{r d}\right|^{2}<g(S N R)\right]
\end{aligned}
$$

Further derivation:

$$
\begin{aligned}
& \frac{1}{g^{2}(S N R)} P_{s d}^{c}= \\
& =\underbrace{\frac{1}{g(S N R)} \operatorname{Pr}\left[\left|\partial_{s r}\right|^{2}<g(S N R)\right.}_{\rightarrow d_{s r}^{\alpha}} \underbrace{\frac{1}{g(S N R)} \operatorname{Pr}\left[2\left|\partial_{s d}\right|^{2}<g(S N R)\right]}_{\rightarrow d_{s d}^{\alpha} / 2} \\
& \quad+\underbrace{\operatorname{Pr}\left[\left|\partial_{s r}\right|^{2} \geq g(S N R)\right.}_{\rightarrow 1} \underbrace{\frac{1}{g^{2}(S N R)} \operatorname{Pr}\left[\left|\partial_{s d}\right|^{2}+\left|\partial_{r d}\right|^{2}<g(S N R)\right]}_{\rightarrow d_{s d}^{\alpha} d_{r d}^{\alpha} / 2} \\
& \rightarrow\left(1 / 2 d_{s d}^{\alpha}\left(d_{s r}^{\alpha}+d_{r d}^{\alpha}\right)\right)
\end{aligned}
$$

So we get the outage probability expression of cooperative communication is:

$$
P_{s d}^{C} \approx \frac{1}{2} d_{s d}^{\alpha}\left(d_{s r}^{\alpha}+d_{r d}^{\alpha}\right) \frac{\left(2^{2 R}-1\right)^{2}}{S N R^{2}}
$$

For the convenience of the numerical simulation and comparison analysis, outage probability expression of information transmission through two-hop is derived on the basis of direct communication between vehicles, in this case, the destination vehicles node can only receive information from the relay vehicle node without the process of collaborative optimization, it is the equivalent of using direct communication for two times to complete the spread of information. Outage probability expressions are derived as follows:

$$
\begin{aligned}
P_{s d}^{T} & =1-\left(1-P_{s r}^{D}\right)\left(1-P_{r d}^{D}\right) \\
& \approx d_{s r}^{\alpha} \frac{\left(2^{R}-1\right)}{S N R}+\left(1-d_{s r}^{\alpha} \frac{\left(2^{R}-1\right)}{S N R}\right) d_{r d}^{\alpha} \frac{\left(2^{R}-1\right)}{S N R}
\end{aligned}
$$

Figure 2 is the simulation diagram of the outage probability of communication between vehicles when $\alpha=4, R=1 \mathrm{bits} / \mathrm{s} / \mathrm{Hz}$, and the normalized distance $d_{s d}=1, d_{s r}=d_{r d}=\frac{1}{2} d_{s d}$, the vertical axis is the outage probability value, the horizontal axis is the signal-to-noise ratio. The diagram shows two-hop communication outage probability is slightly lower than direct communication, cooperative communication outage probability signifiantly lower than the other two kinds of communication modes, and the reliability of communication is obviously advantaged when the SNR rises. 


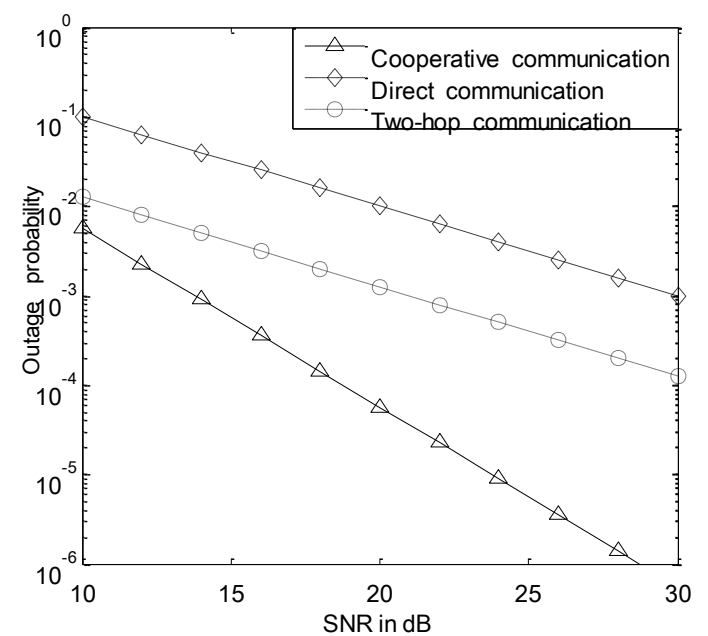

Figure 2. The outage probability of the three kinds of communication

\section{B. Transmission Power of Cooperative Transmission}

The distance from relay vehicle node $r$ to the source vehicle node $s$ and destination vehicle node $d$ is one of the important parameters which influences the performance of collaborative communication[8], so we assume thant the node $\mathrm{r}$ is at the middle point of the node $\mathrm{s}$ and $\mathrm{d}$ firstly, that is $d_{s r}=d_{r d}=\frac{1}{2} d_{s d}$, and then extend it to any position, and get the simulation analysis .

Define global minimum transmission power between vehicles of cooperative communication is as:

$$
W_{s d}^{C}=2 S N R \times N_{0} R B
$$

Due to (11) and (13), we can get:

$$
W_{s d}^{C}=N_{0} R B \frac{d_{s d}^{\alpha}\left(2^{2 R}-1\right)}{2^{\alpha / 2} \sqrt{P_{s d}}}
$$

Where $B$ is the assigned bandwidth, $P_{s d}$ is the outage probability, $N_{0}$ is the noise power spectral density.

Using the same method we can conclude the minimum transmission power of direct communication between vehicles as:

$$
\begin{aligned}
W_{s d}^{D} & =S N R \times N_{0} R B \\
& =N_{0} R B \frac{d_{s d}^{\alpha}\left(2^{R}-1\right)}{P_{s d}}
\end{aligned}
$$

Comparing the transmission power of both cases in ratio method as:

$$
\begin{aligned}
\frac{W_{s d}^{C}}{W_{s d}^{D}} & =\frac{2 d_{s d}^{\alpha}\left(2^{2 R}-1\right)}{2^{\alpha / 2} \sqrt{P_{s d}}} / \frac{d_{s d}^{\alpha}\left(2^{R}-1\right)}{P_{s d}} \\
& =\frac{\left(2^{R}-1\right)}{2^{\alpha / 2-1}} \sqrt{P_{s d}}
\end{aligned}
$$

(16) shows that the data transfer rate $R$ and outage probability $P_{s d}$ determine the performance of collaborative communication between vehicles which has obvious advantages compared to direct communication in terms of power. when data rate is moderate and outage probability is small, the value of equation (16) will be much less than 1 , we can see collaborative communication between vehicles has obvious advantages in terms of power performance.
Put $d_{s r}=d_{r d}=\frac{1}{2} d_{s d}$ into (12), we can get:

$$
P_{s d}=d_{s r}^{\alpha} \frac{\left(2^{R}-1\right)}{S N R}+d_{r d}^{\alpha} \frac{\left(2^{R}-1\right)}{S N R}-d_{s r}^{\alpha} d_{r d}^{\alpha} \frac{\left(2^{R}-1\right)^{2}}{S N R^{2}}
$$

Using the ditto method we can conclude the transmission power of direct communication with two-hop as[9]:

$$
\begin{aligned}
W_{s d}^{T} & =2 S N R \times N_{0} R B \\
& =N_{0} R B \frac{d_{s d}^{\alpha}\left(2^{R}-1\right)\left(1+\sqrt{1-P_{s d}}\right)}{2^{(\alpha-1)} \sqrt{P_{s d}}}
\end{aligned}
$$

Comparing the transmission power of two direct communication in ratio method as:

$$
\frac{W_{s d}^{T}}{W_{s d}^{D}}=\frac{1+\sqrt{1-P_{s d}}}{2^{\alpha-1}} \approx \frac{2-\frac{1}{2} P_{s d}}{2^{\alpha-1}}
$$

(19)shows that two-hop communication has higher energy efficiency when the value of $P_{s d}$ much less than 1 in gener and the value of $\alpha$ is not less than 4 . then we extend the relay vehicle node to any position, and get the simulation analysis

Assume the relay node $r$ located anywhere between the source node $\mathrm{s}$ and the destination node $\mathrm{d}$, and assume that the outage probability $P_{s d}=0.01$, and $\alpha=4$, $R=1 \mathrm{bits} / \mathrm{s} / \mathrm{Hz}$ the communication power of direct communication can be derived by the formula (15) , and the normalized transmission power consumption $W=W_{s d}^{D} / R B N_{0}=100$

In order to study the power consumption of two-hop communication and collaboration communications, we place relay vehicle node $r$ into a two-dimensional circle plane[10], the range of horizontal and vertical coordinates are $(-0.5,0.5)$, the coordinates $(0,0)$ is the center of the circle plane, each diameter of the circle plane can be seen as a communication path and its length is normalized distance 1 , a relay vehicle node $r$ can be located anywhere of the circle plane, and use the normalized transmission power consumption $W=W_{s d} / R B N_{0}$ mean $\mathrm{z}$ axis.

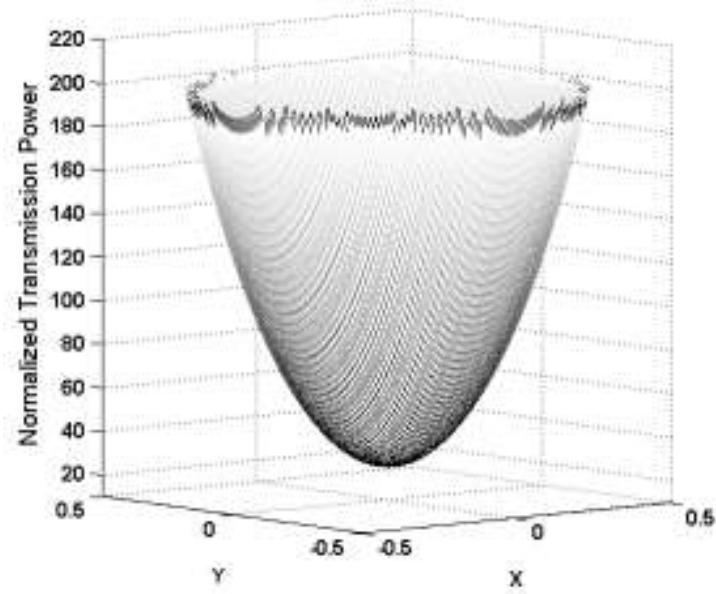

Figure 3. Communication power of two-hop transmission

Figure 3 is the transmission power loss of the two-hop communication when the relay vehicle nodes in different locations, the maximum normalized power is 200 , the 
middle point is the lowest value location which is 25 . Figure 4 is the transmission power loss of the cooperative communication when the relay vehicle nodes in different locations, the maximum normalized power is 42.4 , the middle point is the lowest value location which is 15 .

The figure shows the location where these two communication transmission powers obtain the optimal solution is the center of the circular plane, and since the symmetry of position, the relay node which in the same circle have the same power value. The simulation comparison shows that the performance of cooperative communication are significantly better than the two-hop communication and direct communication at each location in terms of power consumption.

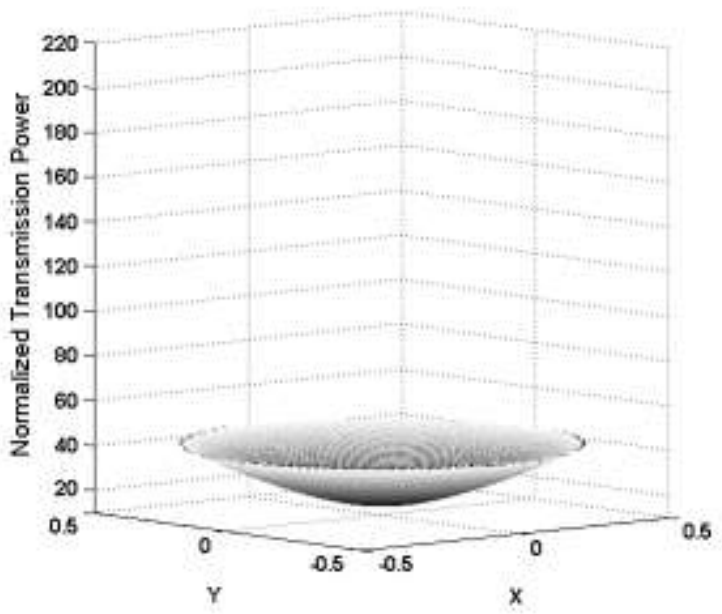

Figure 4. Communication power of cooperative transmission

\section{CONCLUSION}

This paper studied the transmission performance of cooperative communication based on cross-layer routing design in the wireless vehicle Ad-hoc networks, the crosslayer cooperative transmission model is built, and then according to different quality of service requirements, The objective functions expressions of the optimal solutions for two addressed optimization problems are developed. Simulation results show that the criteria using cooperative transmission is typically more efficient than the traditional schemes in terms of end-to-end reliability and total transmission power.

\section{ACKNOWLEDGMENT}

This research was supported by the National Natural Science Foundation of China (No. 61371113) and the Applied Basic Research Project of Ministry of Transport (No. 2013-319-825-110).

\section{REFERENCES}

[1] H. Hartenstein and K. P. Laberteaux, "A tutorial survey on vehicular ad hoc networks," in IEEE Commun. Mag., vol. 46, pp.164-171, 2008.

[2] U. Lee and M. Gerla, "A survey of urban vehicular sensing platforms,'Elsevier Computer Networks, vol. 54, no. 4, pp. 527544, Mar. 2010.

[3] C.-X. Wang, X. Hong, X. Ge, X. Cheng, G. Zhang, and J. S Thompson, "Cooperative mimo channel models: a survey," IEEE Commun. Mag.,vol. 48, pp. 80-87, Feb. 2010.
[4] J. Nzouonta, N. Rajgure, G. Wang, and C. Borcea, "Vanet routing on city roads using real-time vehicular traffic information," IEEE Trans.Veh. Technol., vol. 58, pp. 609-3626, July. 2009.

[5] E. Hossain, G. Chow, and V.C.M. Leung, "Vehicular Telematics over Heterogeneous Wireless Networks: A Survey," Computer Communications, vol. 33, Issue 7, pp. 775-793, May .2010.

[6] W.-L. Jin and W. Recker, "An analytical model of multihop connectivity of intervehicle communication systems," IEEE Trans. Wireless Commun, vol. 9, pp. 106-112, January .2010.

[7] H. T. Chang, H. Shan, and W. Zhuang, "Infotainment and road safety service support in vehicular networking: From a communication perspective" Mechanical Systems and Signal Processing, vol 25, Issue 6, pp. 2020-2038, November .2010.

[8] G. Ferrari, M. Martal'o, and R. Pagliari, "Decentralized detection in clustered sensor networks," IEEE Trans. Aerosp. Electron. Syst., vol. 47, no. 2, pp. 959-973, Apr. 2011.

[9] M. Gerla and L. kleinrock, "Vehicular Networks and future of the mobile internet" Computer Networks, vol 55, Issue 2, pp. 457-469, February 2011.

[10] C. Suthaputchakun, and Z. Sun, "Priority based Routing Protocol in Vehicular Ad hoc Network", the 16th IEEE Symposium on Computers and Communications, Greece, 2011, pp.723-728. 